\title{
FIRST ORDER PROPERTIES OF PAIRS OF CARDINALS
}

\author{
BY H. JEROME KEISLER
}

Communicated by D. Scott, September 23, 1965

We consider models of a countable first order logic $L$ with an identity symbol and predicate symbols $U, P_{0}, P_{1}, \cdots, U$ being unary. A model $\mathfrak{U}=\left\langle A, U_{\mathfrak{R}}, P_{0 \mathfrak{P}}, \cdots\right\rangle$ for $L$ is said to be a twocardinal model if $A$ is infinite and the power of $U_{\mathfrak{Q}}$ is less than the power of $A$. By a set of axioms for two-cardinal models we mean a set $\Sigma$ of sentences of $L$ such that $\mathfrak{A}$ is a model of $\Sigma$ if and only if there exists a two-cardinal model which is elementarily equivalent to $\mathfrak{A}$. Using results of Fuhrken [1], Vaught [4] proved the following theorem.

THEOREM (VAUGHT). There is a set of axioms for two-cardinal models. If the language $L$ is recursive, then there is a recursive set of axioms for two-cardinal models.

We say that $L$ is recursive if the number of argument places of the symbol $P_{n}$ is a recursive function of $n$. Vaught's proof depends on the fact that if $\Sigma^{*}$ is a recursive set of sentences in an extension $L^{*}$ of the language $L$, then there is a recursive set $\Sigma$ of sentences of $L$ such that $\Sigma$ and $\Sigma^{*}$ have exactly the same consequences in $L$. In principle his proof can be used to construct a particular set of axioms for two-cardinal models, but the set seems to be so complicated that in practice one cannot easily tell whether or not a given sentence belongs to it. Vaught has proposed the problem of finding a simple set of axioms for two-cardinal models. The author heard about Vaught's problem through Dana Scott.

In this note we shall give a particular set of axioms for two-cardinal models which is simple enough to be written down as a fairly short axiom scheme. Our theorem was stated without proof in [2]. Let the individual variables of $L$ be $v_{i}, x_{i}, y_{i}, z_{i}$, where $i=0,1,2, \cdots$.

THEOREM 1. A set of axioms for two-cardinal models is given by the set $\Gamma$ of all sentences of the form

$$
\begin{gathered}
\exists v_{0} \forall x_{0} \exists y_{0} Z_{0} \cdots \forall x_{n} \exists y_{n} z_{n} \\
{\left[\bigwedge_{i=0}^{n} v_{0} \neq y_{i} \& \bigwedge_{i, j=0}^{n}\left(U\left(x_{j}\right) \& x_{i}=z_{j} \rightarrow y_{i}=x_{j}\right)\right.} \\
\left.\& \bigwedge_{j=0}^{m}\left(\phi_{j}\left(x_{0}, \cdots, x_{n}\right) \rightarrow \phi_{j}\left(y_{0}, \cdots, y_{n}\right)\right)\right] .
\end{gathered}
$$


There is one instance of the scheme $\left(^{*}\right)$ for each $n$ and each finite sequence of formulas $\phi_{0}, \cdots, \phi_{m}$ of $L$ with the free variables $x_{0}, \cdots, x_{n}$.

It is obvious that the set $\Gamma$ of sentences is recursive provided that the language $L$ is recursive. To prove Theorem 1, we shall use a lemma of Vaught, which is proved in Morley and Vaught [3, p. 55]. We use the standard notations $\mathfrak{A} \cong \mathfrak{B}, \mathfrak{A} \equiv \mathfrak{B}, \mathfrak{A} \prec \mathfrak{B}$, to mean that $\mathfrak{A}$ is isomorphic to $\mathfrak{B}, \mathfrak{A}$ is elementarily equivalent to $\mathfrak{B}$, and $\mathfrak{A}$ is an elementary submodel of $\mathfrak{B}$ (see, for example, [3]).

Lemma (VAUGhT). For each model $\mathfrak{A}$ for $L$, the following two conditions are equivalent:

(i) There is a two-cardinal model $\mathfrak{B}$ such that $\mathfrak{B} \equiv \mathfrak{A}$.

(ii) There exist countable models $\mathfrak{B}$, $\mathfrak{E}$, such that $\mathfrak{B} \equiv \mathfrak{A}$, $\mathfrak{E} \prec \mathfrak{B}$, $\mathfrak{C} \neq \mathfrak{B}$, $\mathfrak{\complement} \cong \mathfrak{B}$, and $U_{\mathbb{夭}}=U_{\mathfrak{B}}$.

We now prove Theorem 1 . First the easy direction. We let $\mathfrak{A}$ be elementarily equivalent to a two-cardinal model and prove that $\mathfrak{A}$ is a model of $\Gamma$. Consider a sentence $\psi$ of the form $\left({ }^{*}\right)$ in $\Gamma$. Let $\mathfrak{B}$, $\mathcal{C}$ be as in part (ii) of the lemma and let $f$ be an isomorphism from $B$ to ç. For all $\phi_{i}$ and all $b_{0}, \cdots, b_{n} \in B$, the following are equivalent:

$$
\begin{gathered}
b_{0}, \cdots, b_{n} \text { satisfies } \phi_{i} \text { in } \mathfrak{B} ; \\
f b_{0}, \cdots, f b_{n} \text { satisfies } \phi_{i} \text { in } \mathfrak{E} ; \\
f b_{0}, \cdots, f b_{n} \text { satisfies } \phi_{i} \text { in } \mathfrak{B} .
\end{gathered}
$$

We shall use the fact that the first line above implies the third line. To show that $\psi$ holds in $\mathscr{B}$, we find an element $v_{0}$ in $B$ and functions $y_{i}\left(x_{0}, \cdots, x_{i}\right), z_{i}\left(x_{0}, \cdots, x_{i}\right), i=0, \cdots, n$ on $B$ such that the inner part of $\psi$ holds in $\mathscr{B}$ for all $x_{0}, \cdots, x_{n}$ in $B$. Take for $v_{0}$ any element of $B-C$. Let $y_{i}\left(x_{0}, \cdots, x_{i}\right)=f\left(x_{i}\right)$. If $U\left(x_{i}\right)$, let $z_{i}\left(x_{0}, \cdots, x_{i}\right)$ $=f^{-1}\left(x_{i}\right)$, and otherwise choose $z_{i}$ arbitrarily. These choices of $v_{0}, y_{i}$, $\boldsymbol{z}_{\boldsymbol{i}}$ show that $\psi$ holds in $\mathfrak{B}$ and thus in $\mathfrak{A}$. Therefore $\mathfrak{A}$ is a model of $\psi$.

We now prove the converse. Assume $\mathfrak{A}$ is a model of $\Gamma$. We extend the language $L$ to a language $L^{*}$ by adding a new individual constant $c$ and function symbols $F_{n}, G_{n}$ with $n+1$ argument places, $n=0,1$, $2, \ldots$ Let $\Gamma^{*}$ be the set of all the sentences below:

(1) $\forall x_{0} \cdots x_{n}, c \neq F_{n}\left(x_{0}, \cdots, x_{n}\right)$.

(2) $\forall x_{0} \cdots x_{n},\left(U\left(x_{j}\right) \& x_{i}=G_{j}\left(x_{0}, \cdots, x_{j}\right) \rightarrow F_{i}\left(x_{0}, \cdots, x_{i}\right)=x_{j}\right)$.

(3) $\forall x_{0} \cdots x_{n},\left[\phi\left(x_{0}, \cdots, x_{n}\right) \rightarrow \phi\left(F_{0}\left(x_{0}\right), \cdots, F_{n}\left(x_{0}, \cdots, x_{n}\right)\right)\right]$.

The scheme (1) contains one sentence for each $n$, (2) contains a sentence for each $n$ and each $i, j \leqq n$, while (3) contains one sentence for each $n$ and each formula $\phi\left(x_{0}, \cdots, x_{n}\right)$ of the original language $L$. Since $\mathfrak{A}$ is a model of $\Gamma$, it follows that for each finite subset $\Gamma_{0}^{*} \subset \Gamma^{*}$ 
the model $\mathfrak{A}$ can be expanded to a model ( $\mathfrak{A}, c, F_{0}, \cdots, G_{0}, \cdots$ ) of $\Gamma_{0}^{*}$. Let $\Delta$ be the set of all sentences of $L$ which hold in $\mathscr{A}$. Then the set of sentences $\Delta \cup \Gamma^{*}$ is finitely satisfiable. By the compactness and Löwenheim-Skolem theorems, $\Delta \cup \Gamma^{*}$ has a countable model $\left(\mathfrak{B}, c, F_{0}, \cdots, G_{0}, \cdots\right)$. Since $\mathfrak{B}$ is a model of $\Delta, \mathfrak{B} \equiv \mathfrak{A}$. We shall show that $\mathfrak{B}$ has the property described in part (ii) of the lemma.

Let us list the elements of $B$, say $B=\left\{b_{0}, b_{1}, \cdots, b_{n}, \cdots\right\}$. Define the function $f$ on $B$ into $B$ by

$$
f\left(b_{n}\right)=F_{n}\left(b_{0}, b_{1}, \cdots, b_{n}\right) .
$$

This definition is unambiguous even if some $b$ occurs more than once in the sequence $b_{0}, b_{1}, \cdots$, because of (3). We claim that $f$ has the following three properties:

(4) Range of $f \neq B$.

(5) $U_{\mathfrak{B}}$ Crange of $f$.

(6) For all formulas $\phi\left(x_{0}, \cdots, x_{n}\right)$ of $L$, if $b_{0}, \cdots, b_{n}$ satisfies $\phi$ in $\mathscr{B}$ then so does $f b_{0}, \cdots, f b_{n}$.

Condition (4) is guaranteed by the sentences (1). Condition (5) is guaranteed by (2), because if $U\left(b_{j}\right)$ and $b_{i}=G_{j}\left(b_{0}, \cdots, b_{j}\right)$, then choosing $n \geqq i, j$ we have $f\left(b_{i}\right)=F_{i}\left(b_{0}, \cdots, b_{i}\right)=b_{j}$. Finally, condition (6) is guaranteed by (3).

Now let $\mathbb{C}$ be the submodel of $B$ such that $C$ is the range of $f$. It follows from (4) that $\mathbb{C} \neq \mathfrak{B}$, and from (5) that $U_{\mathfrak{B}} \subset C$. From (6) we see that $f$ is an isomorphism from $\mathfrak{B}$ to $\mathbb{C}$, and it follows that $U_{\mathfrak{C}}=U_{\mathfrak{B}}$ and $\mathfrak{B} \cong \mathbb{C}$. It also follows from (6) that $\mathfrak{E} \prec \mathfrak{B}$, because if $f b_{0}, \cdots, f b_{n}$ satisfies $\phi$ in $\mathfrak{C}$ then $b_{0}, \cdots, b_{n}$ satisfies $\phi$ in $\mathfrak{B}$ and hence $f b_{0}, \cdots, f b_{n}$ satisfies $\phi$ in $\mathfrak{B}$. By the lemma, there is a two-cadinal model which is equivalent to $\mathfrak{A}$. Our proof is complete.

There are several ways in which we can modify the scheme $\left(^{*}\right)$ without affecting the proof of Theorem 1 . This gives us some other slightly different sets of axioms for two-cardinal models. One possibility is to replace the scheme $(*)$ by

$$
\begin{gathered}
\exists v_{0} \forall x_{0} \exists y_{0} z_{0} \cdots \forall x_{n} \exists y_{n} z_{n} \\
{\left[\bigwedge_{i=0}^{n} v_{0} \neq y_{i} \& \bigwedge_{i, j=0}^{n}\left(U\left(x_{j}\right) \rightarrow\left(x_{i}=z_{j} \leftrightarrow y_{i}=x_{j}\right)\right)\right.} \\
\left.\& \bigwedge_{j=0}^{m}\left(\phi_{j}\left(x_{0}, \cdots, x_{n}\right) \leftrightarrow \phi_{j}\left(y_{0}, \cdots, y_{n}\right)\right)\right] .
\end{gathered}
$$

Another scheme of axioms for two-cardinal models which will work with the same proof is: 
$(* * *)$

$$
\begin{aligned}
& \exists v_{0} \forall x_{0} w_{0} \exists y_{0} z_{0} \cdots \forall x_{n} w_{n} \exists y_{n} z_{n} \\
& {\left[\bigwedge_{i=0}^{n} v_{0} \neq y_{i} \&\left[\left(\neg \exists v_{1} U\left(v_{1}\right) \vee \bigwedge_{i=1}^{n} U\left(w_{i}\right)\right)\right.\right.} \\
& \\
& \rightarrow \bigwedge_{j=0}^{m}\left(\phi_{j}\left(x_{0}, \cdots, x_{n}, z_{0}, \cdots, z_{n}\right)\right. \\
& \left.\left.\left.\leftrightarrow \phi_{j}\left(y_{0}, \cdots, y_{n}, w_{0}, \cdots, w_{n}\right)\right)\right]\right] .
\end{aligned}
$$

Everything works out just as well if we define the notion of a twocardinal model in the following slightly different way. Let the language $L$ have two unary predicates $U, V$, in addition to $P_{0}, P_{1}, \cdots$. By a two-cardinal model we now mean a model $\mathfrak{A}$ for $L$ such that $V_{\mathfrak{A}}$ is infinite and the power of $U_{\mathfrak{A}}$ is less than the power of $V_{\mathfrak{Q}}$. Then we get a set of axioms for two-cardinal models simply by adding the extra term $V\left(v_{0}\right)$ to the conjunction inside the quantifiers in the scheme $\left(^{*}\right)$.

\section{REFERENCES}

1. G. Fuhrken, Skolem-type normal forms for first-order languages with a generalized quantifier, Fund. Math. 54 (1964), 291-302.

2. H. J. Keisler, First order properties of pairs of cardinals, Abstract 65 T-291, Notices Amer. Math. Soc. 12 (1965), 482.

3. M. Morley and R. Vaught, Homogeneous universal models, Math. Scand. 11 (1962), 37-57.

4. $\mathrm{R}$. Vaught, The completeness of logic with the added quantifier "there are uncountably many," Fund. Math. 54 (1964), 303-304.

UNIVERSITY OF WISCONSIN 